\title{
Anxiety and ASD: Current Progress and Ongoing Challenges
}

\author{
Mikle South $^{1} \cdot$ Jacqui Rodgers $^{2}$ (D) Amy Van Hecke ${ }^{3}$
}

Published online: 7 October 2017

(C) Springer Science+Business Media, LLC 2017

\begin{abstract}
Symptoms of anxiety add significant burden to many autistic individuals and their loved ones. There is an urgent need for better understanding of the unique underlying mechanisms of anxiety in ASD, and for the development of more specific assessment methods and treatment recommendations. This special issue brings together 24 articles grouped into three themes; mechanisms, measurement, and intervention. The result is a review of current anxiety research in ASD that is both broad and deep. Key themes include recognition of the importance individual differences in aetiology and presentation of anxiety in ASD, the need for a more nuanced understanding of the interactions between anxiety and characteristics of ASD and the need to develop appropriately adapted treatments.
\end{abstract}

This special issue of the Journal of Autism and Developmental Disorders (JADD) aims to showcase the most recent research on anxiety in ASD. Around 50\% of autistic individuals will experience anxiety that significantly impacts on their daily lives and the lives of their loved ones. When present, anxiety reduces quality of life and interferes with education, employment, and achievement of potential. The urgent need to address this pressing clinical issue was highlighted by the Autistica Priority Setting Partnership (2015), undertaken in collaboration with the autism community in the UK, which identified mental health concerns as the top priority for autism research with specific emphasis on the

Jacqui Rodgers

jacqui.rodgers@newcastle.ac.uk

1 Brigham Young University, Provo, USA

2 Newcastle University, Newcastle upon Tyne, UK

3 Marquette University, Milwaukee, USA development of interventions to reduce anxiety identified as amongst the top five research priorities.

The idea for this Special Issue arose as a consequence of discussions which took place at the Anxiety and ASD Special Interest Group at the International Meeting for Autism Research in 2015, with the call for papers issued in summer of 2015. We have been privileged to receive very many high quality submissions, all of which have been subjected to standard peer review processes. The outcome of this endeavour is this special issue, comprising 24 excellent papers from the leading international researchers in the field. Our aspiration was to present to JADD readership the most up-to-date scientific and methodological developments in the field. The papers included in this special issue fall into three broad themes: (1) mechanisms and correlates underpinning the development and maintenance of anxiety in ASD, (2) issues with measurement, assessment, and stability of anxiety in ASD, and (3) interventions for anxiety for autistic people.

\section{Mechanisms, Measurement, and Application to Treatment: The Special Issue}

The first theme concerns mechanisms and correlates of anxiety in ASD. Herrington and colleagues identified decreased right amygdala volume in a relatively large sample of children diagnosed both with autism and an anxiety disorder, compared to a group of autistic children with no anxiety disorder. An important take-away from the study is that a onesize-fits-all approach for treatment of anxiety and related concerns is unlikely to be helpful. Turning to physiological mechanisms and threat responsivity, South et al. found that autistic children showed lower skin conductance activity to a potentially threatening stranger; skin conductance activity was related to anxiety symptom severity and also 
to movement confounds, highlighting the need for precision of measurement. A diminished threat response fits with data presented by Grossman and colleagues, who examined state versus trait anxiety in autistic adolescents, finding that higher trait anxiety in ASD may contribute to dampened physiological stress responses. Finally, research by Milosavlejevic et al. found expected high levels of anxiety in autistic children, but also found that threat sensitivity may present more strongly in siblings of autistic children versus children who meet criteria for ASD themselves, perhaps pointing to a familial mechanism linking anxiety and ASD.

Studies of anxiety across neurodevelopmental conditions are important for establishing areas of overlap and difference that contribute to understanding of underlying cause and the development of targeted intervention strategies. A group of papers in this issue discuss the specificity of anxiety and its relation to ASD versus other aetiologies: Crawford et al. examine ASD and Fragile X, Cornelia de Lange, and Rubinstein-Taybi syndromes; Roberts et al. examine ASD, Fragile $\mathrm{X}$, and anxiety in pre-schoolers; Factor et al. and Schiltz et al. examine ASD and ADHD; and finally, Smith-Johnston and colleagues consider clinical depression as a factor linked to social competence in ASD. Royston et al. explicitly examined the possible contribution of intellectual disability (ID) to anxiety: their meta-analysis concluded that risk for anxiety in Williams Syndrome was four times greater than the risk arising from ID alone.

A final group of papers for this theme focus on correlated symptoms and concerns that may contribute to anxiety in ASD: Joyce et al. examine intolerance of uncertainty as both an indicator and contributor to symptoms of ASD and patterns of anxiety; Capriola et al. and Kleberg et al. focus on social challenges, including fear of negative evaluation and orienting to eyes, respectively, as mechanisms underlying social anxiety in ASD; and, Oszivadjian and colleagues find that anxious imagery is even more prevalent in ASD than in children with anxiety alone. These papers collectively reinforce the importance of considering very specific mechanisms of anxiety in planning treatment across individuals.

Measurement, assessment, and stability make up the second theme. Papers in this group include Glod et al.'s and Jitlina et al.'s examination of psychometric properties of the Spence Children's Anxiety Scale, with both papers suggesting areas of needed improvement. Finally, both Magiati et al. and Schiltz et al. find stability over time for presentation of anxiety symptoms in autism. As new measures of anxiety that have been developed specifically for autism become available (for example, see Kerns et al. 2017; Rodgers et al. 2016) it will be vital to sort out how typical and atypical anxiety interact across neurodevelopmental conditions and within individuals.

The final theme considers issues related to treatment of anxiety in ASD. Clarke and colleagues, and Kovshoff and colleagues, present promising results of randomized controlled trials of Attwood's (2004) Exploring Feelings schoolbased cognitive behavioural therapy (CBT) for anxiety in autistic children. Drmic and colleagues present similarly promising results for a school-based adaptation of the Facing Your Fears (Reaven et al. 2012) intervention for autistic adolescents.. Maddox et al. present impactful research on the durability of CBT over time, as well as predictive concomitants. Moskowitz and colleagues present a multiple baseline trial of a positive behaviour and CBT intervention for anxiety in autistic children with ID, showing promising results on both behavioural and psychophysiological measures. Keefer and colleagues examined how intolerance of uncertainty (IU) poses additional challenges when delivering adapted CBT: children with high IU scores at baseline benefitted less from a standard approach. They suggest that children with high levels of IU—with or without autism-may benefit from targeted intervention as part of CBT. This idea is supported by the innovative intervention developed by Rodgers and colleagues, which specially focused on assisting parents in handling challenges with IU in their autistic children.

\section{Broader and Deeper: Blueprints for Future Research}

We believe that the research presented here is an exciting overview of the field. There remains, however, much still to be done and many unanswered questions. Of note is the paucity of research on anxiety in autistic adults, or those with comorbid intellectual disability. Investigation of potential gender differences in emotion awareness and coping response will be critical for accumulating research on gender differences in ASD symptom presentation, diagnosis, and treatment response (McVey et al. 2017; Pisula et al. 2017). One critical question not answered by existing studies is how brain and physiological responses may be different for autistic people who present with symptoms of anxiety that do not fit traditional criteria for anxiety disorders, including what Kerns and colleagues (2014) have labeled as "atypical" anxiety.

From a methodological standpoint, there has been a heavy reliance on self or proxy report questionnaires, often utilising tools which have not been developed for or validated with autistic people. Understanding how questionnaire data and physiological data can inform each other has been difficult for anxiety researchers generally, perhaps because of faulty conceptual frameworks (LeDoux and Pine 2016). This may be an especially problematic task in the context of ASD. The use of multi-trait, multi-method study designs is essential, but so is the need for flexible, innovative frameworks of how brain and behaviour interact in anxiety and ASD. Much 
of the existing work is cross-sectional, significantly impacting on our understanding of the developmental trajectory of anxiety in autism.

The inclusion of atypical sensory processing in the diagnostic criteria for autism is new with DSM-5, but has a long history of clinical relevance (Leekam et al. 2007; O'Neill and Jones 1997). There is now growing evidence for links between sensory function and anxiety along with autism core symptoms including repetitive behaviour and social competence (Beaumont et al. 2015; South and Rodgers 2017). Future research including questionnaire, physiological and imaging paradigms will be useful for clarifying underlying mechanisms that link these important constructs together. Treatment regimens that integrate sensory regulation, emotion regulation, and social skills training are an important avenue for study.

We give thanks to the International Society for Autism Research for sponsoring the IMFAR special interest group that got this ball rolling, and to JADD editorial and production staff for their support of the project. We hope that the canon of work presented in this Special Issue of JADD will serve as a further call to arms for current and future autism researchers to continue to pursue these and other important clinical questions.

Author Contributions MS, JR and AVH contributed equally to all aspects of this manuscript.

\section{Compliance with Ethical Standards}

Conflict of interest None of the authors have any conflicts of interest.

Research Involving Human and Animal Participants This article does not contain any studies with human participants or animals performed by any of the authors.

\section{References}

Attwood, T. (2004). Exploring Feelings: Anxiety. Cognitive Behaviour Therapy to Manage Anxiety. Future Horizons.

Beaumont, R., Rotolone, C., \& Sofronoff, K. (2015). The Secret Agent Society social skills program for children with high-functioning autism spectrum disorders: A comparison of two school variants. Psychology in the Schools, 52(4), 390-402. doi:10.1002/ pits. 21831.

James Lind Alliance \& Autistica Research Priority Setting Partnership. (2015). http://www.jla.nihr.ac.uk/priority-settingpartnerships/ autism/downloads/Autism\%20PSP\%20final\%20report.pdf.

Kerns, C. M., Kendall, P. C., Berry, L., Souders, M. C., Franklin, M. E., Schultz, R. T., ... Herrington, J. (2014). Traditional and atypical presentations of anxiety in youth with autism spectrum disorder. Journal of Autism and Developmental Disorders, 44(11), 2851-2861. doi:10.1007/s10803-014-2141-7.

Kerns, C. M., Renno, P., Kendall, P. C., Wood J. J., \& Storch, E. A. (2017). Anxiety Disorders Interview Schedule-Autism Addendum: Reliability and Validity in Children With Autism Spectrum Disorder. Journal of Clinical Child \& Adolescent Psychology, 46,1 .

LeDoux, J. E., \& Pine, D. S. (2016). Using neuroscience to help understand fear and anxiety: A two-system framework. American Journal of Psychiatry, 173(11), 1083-1093. doi:10.1176/appi. ajp.2016.16030353.

Leekam, S. R., Nieto, C., Libby, S. J., Wing, L., \& Gould, J. (2007). Describing the sensory abnormalities of children and adults with autism. Journal of Autism and Developmental Disorders, 37(5), 894-910. doi:10.1007/s10803-006-0218-7.

McVey, A. J., Schiltz, H., Haendel, A., Dolan, B. K., Willar, K. S., Pleiss, S., ... Van Hecke, A. V. (2017). Brief report: Does gender matter in intervention for ASD? Examining the impact of the PEERS ${ }^{\circledR}$ social skills intervention on social behavior among females with ASD. Journal of Autism and Developmental Disorders, 47(7), 2282-2289. doi:10.1007/s10803-017-3121-5.

O’Neill, M., \& Jones, R. S. P. (1997). Sensory-perceptual abnormalities in autism: A case for more research? Journal of Autism and Developmental Disorders, 27(3), 283-293. doi:10.102 3/A:1025850431170.

Pisula, E., Pudło, M., Słowińska, M., Kawa, R., Strząska, M., Banasiak, A., \& Wolańczyk, T. (2017). Behavioral and emotional problems in high-functioning girls and boys with autism spectrum disorders: Parents' reports and adolescents' self-reports. Autism, 21(6), 738-748. doi:10.1177/1362361316675119.

Reaven, J., Blakeley-Smith, A., Leuthe, E., Moody, E., \& Hepburn, S. (2012). Facing your fears in adolescence: Cognitive-behavioral therapy for high-functioning autism spectrum disorders and anxiety. Autism Research and Treatment. doi:10.1155/2012/423905.

Rodgers, J., Wigham, S., McConachie, H., Freeston M., Honey, E., \& Parr, J. R. (2016). Development of the Anxiety Scale for Children with autism spectrum disorder (ASC-ASD). Autism Research, 9(11), 1205-1215.

South, M., \& Rodgers, J. (2017). Sensory, emotional and cognitive contributions to anxiety in autism spectrum disorders. Frontiers in Human Neuroscience. doi:10.3389/fnhum.2017.00020. 